\title{
The effects of delay of reward on negative contrast effects associated with reductions in reward magnitude
}

\author{
JOHN N. MOORE and JAMES H. McHOSE \\ Southern Illinois University-Carbondale, Carbondale, Illinois 62901
}

\begin{abstract}
The results of three runway experiments in which rats received a reduction in the magnitude of delayed vs. immediate reward are reported. The results indicated that the negative contrast effect associated with a reduction in reward amount is more pronounced when all rewards are delayed.
\end{abstract}

In the past three decades. considerable theoretical attention has focused on the behavioral effects of changes in magnitude of reward in instrumental conditioning. Until quite recently, it appeared that while decreases in reward magnitude produced contrast effects. comparable increases in reward did not (cf. Black. 1968; Dunham. 1968). Thus, rats shifted from large to small reward typically show negative contrast effects (NCEs), depressed performance to small reward as compared with that of subjects trained and maintained on small reward. while subjects shifted from small to large reward do not display positive contrast effects (PCEs). facilitation of performance to large reward as compared with that of subjects maintained on large reward. Recent studies. however, have clearly shown that PCEs attend increases in reward amount if all rewards are delayed following the instrumental response (Mellgren, 1972).

While it is clear that reward delay interacts with increases in reward magnitude such that PCEs are larger and more reliable under delayed as compared with immediate reward conditions, the theoretical basis of this interactive effect remains largely unexplored. Most frequently, the interactive effect has been attributed to the operation of a physiological or psychological "ceiling effect" on the performance of unshifted large reward (control) subjects (cf. Bower. 1961: Mellgren. 1972; Shanab \& Cavallaro. 1975). According to the ceiling hypothesis, PCEs are not observed under conditions of immediate reward because control subjects have achieved optimal response levels: lowering the performance level of control subjects. through delay of reinforcement. provides a context in which the facilitative effects of increases in reward magnitude may be seen. It should be clear that such a hypothesis does not attempt to

This research was supported by grants from the College of Liberal Arts. the Office of Researc.i and Projects. Southern Illinois Lniversity. Carbondale, and an NIMH training grant. General Experimental Psychology. MH 07817-13. Reprint requests should be addressed to James $\mathrm{H}$. McHose. Department of Psychology. Southern Illinois University. Carbondale. Illinois 62901. derive the interactive effects of reward delay and increases in reward amount from any general theoretical treatment of the effects of change in the parameters of reinforcement on instrumental responding.

Several of the theoretical viewpoints relevant to the effects of change in reward magnitude can easily be moditied to incorporate the interactive effects between delay and reward increase. For example, within the framework that PCEs result from perceptual contrast between small and large rewards (e.g.. Bevan \& Adamson. 1960), it could be assumed that delaying rewards enhances perceptual differences. Similarly. for approaches which assume that contrast arises from discrepancies between previously established incentive motivation or expectancy levels and those commensurate with current reinforcement conditions (e.g.. McHose \& Peters. 1975), it could be assumed that delay of reward modifies magnitude-related incentive functions. In either case, such approaches have direct implications for the effects of delay of reinforcement on contrast effects produced by reductions in reward amount. i.e.. NCEs. The present paper reports the results of three studies, each of which provides for an assessment of the effects of reductions in reward amount in delayed as compared with immediate reward conditions.

\section{Subjects \\ METHOD \\ The subjects were 24. 32. and 40 experimentally naive male albino rats. approximately 130.120. and 90 days old. in Experiments 1. 2. and 3, respectively. All subjects were obtained from the Holtzman Company. Madison. Wisconsin.}

\footnotetext{
Apparatus

The apparatus was a straight runway composed of start. run. and goal sections which have been described elsewhere in detail (McHose \& Peters, 1975). A solenoid-operated, side-existing. opaque door separated the start from the run section, which in turn was separated from the goal section by a translucent guillotine retrace door. In Experiments 2 and 3, the goal cup was covered by an opaque solenoid-operated lid which retracted at the end of an experimentally determined interval. The goal cup lid was absent during Experiment 1 . In that experiment, the goal cup was baited via a short metal tube which extended through the ceiling of the
} 


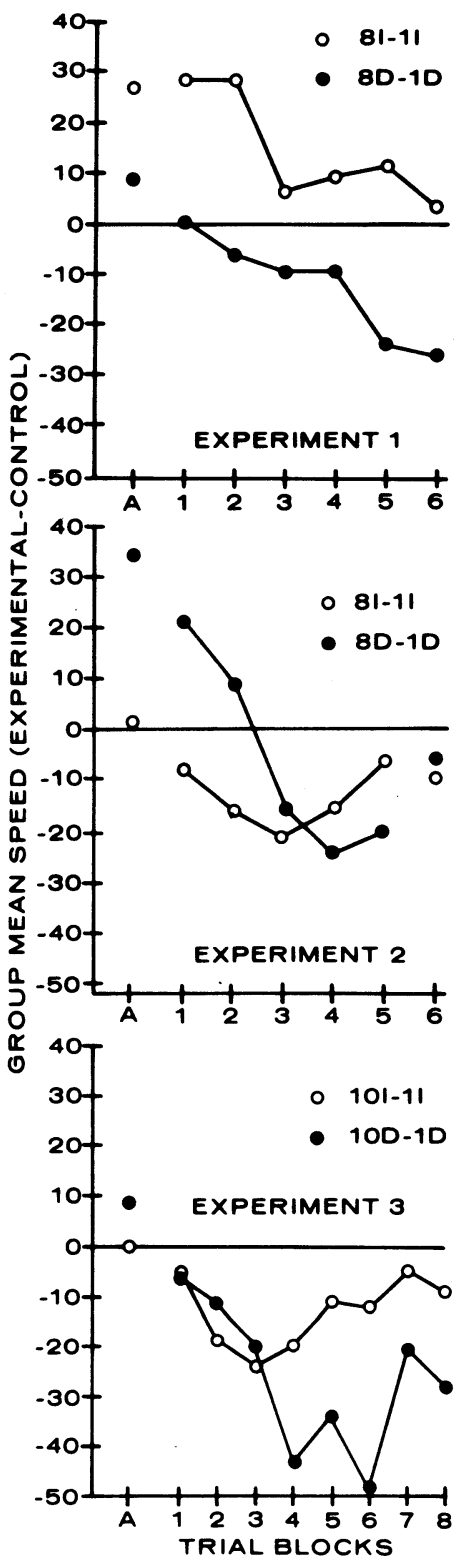

Figure 1. Mean speed differences at the final block of acquisition trials (A) and during postshift.

apparatus and terminated over the goal cup. Photocell-clock circuitry provided response latency measures over four successive segments of the alley.

\section{Procedure}

Procedure prior to the first experimental day consisted of initiation of a food-deprivation schedule, handling, prefeeding, and habituation to the apparatus. Subjects were placed on a 23-h food-deprivation schedule either 10 (Experiment 1) or 14 days (Experiments 2 and 3 ) prior to the first training day. Water was freely available and the schedule was maintained for the duration of the experiment. On each of the last 5 days of the pretraining phase, each subject was handled for approximately $5 \mathrm{~min}$. On the last 3 days prior to the experimental training, the animals were fed approximately $2045-\mathrm{mg}$ Noyes pellets, identical to the subsequent reinforcers, in addition to their daily feeding, and allowed to explore the start and run sections of the apparatus, in pairs, for approximately $5 \mathrm{~min}$.
Subjects were randomly assigned to one of four groups in each experiment, labeled according to the number of pellets received on each trial of the preshift-postshift periods and according to whether rewards were received immediately (I) or delayed $15 \mathrm{sec}$ following a response (D): Groups 8I-1I, 1I-1I, 8D-1D, 1D-1D (Experiments 1 and 2) and Groups 10I-1I, 1I-1I, 10D-1D, 1D-1D (Experiment 3). The experiments differed chiefly with respect to the number of preshift and postshift trials and the distribution of these trials over days. In Experiment 1, all subjects received 24 preshift trials (12 per day) and 18 postshift trials (all administered on the same day). In Experiment 2, subjects received 30 preshift trials (1 on Day ,1, 2 on Day .2. and 3 per day thereafter) and 30 postshift trials (15 per day). In Experiment 3, 20 preshift and 16 postshift trials were administered at the rate of 2 per day. In each experiment, the within-day intertrial interval, in both preshift and postshift periods. was 7-10 min. Subjects were administered trials in running squads. composed of two subjects from each group (two or three subjects in Experiment 3), with each subject receiving its first trial before any subject received its second daily trial, etc.

A running trial was initiated by placing the subject in the start box. After a 3-sec orientation, the startbox door was opened. When the rat entered the goalbox, the retrace door was lowered and, if the subject was in a delay group, a timer was initiated. After the delay period had elapsed, the animal received its prescribed reward. In Experiment 1, this was accomplished by dropping either one or eight food pellets down the metal tube which opened over the goal cup. If an animal was in an immediate group, the goal cup was baited prior to the beginning of the runway trial. In Experiments 2 and 3 , the goal cup was always baited and covered by the goal cup lid prior to placing the subject in the startbox. The lid was retracted immediately following entry into the goalbox for animals in the immediate group or after a $15-\mathrm{sec}$ delay period for subjects in the delay groups. All subjects were removed from the goalbox immediately after consumption of the reward. All latencies were converted to speed scores.

\section{RESULTS}

Since the various response measures yielded similar information, only reciprocated total alley latencies will be presented. Group mean total speed differences as a function of trial blocks are presented in Figure 1. The performance level of each experimental (reward reduction) condition is plotted relative to that of the appropriate control condition for the last acquisition (A) trial block and for successive postshift trial blocks. In Experiments 1 and 2, each postshift block contains the data for three trials, with the exception of Block 6 in Experiment 2, which contains data for 15 trials. Each postshift trial block in Experiment 3 contains the data for two trials.

Turning first to the data of primary interest, the postshift data, it may be seen in Figure 1 that in each experiment reduction in the amount of delayed reward yielded a more pronounced depression of postshift performance than did a comparable reduction in the amount of immediate reward. Analysis of variance of the postshift data yielded a reliable Delay by (Preshift) Magnitude interaction over Blocks 5-6 in Experiment $1(\mathrm{~F}=5.15$, $\mathrm{df}=$ $1 / 20, p<.05)$, and over Blocks 5-6 in Experiment 3 $(\mathrm{F}=7.42, \mathrm{df}=1 / 36, \mathrm{p}<.01)$. Post hoc comparisons indicated that, in Experiment 1, Group 8D-1D ran reliably slower than Group 1D-1D $(F=6.30, d f=1 / 20, p<.05)$, while no differences 
were obtained between Groups 8I-1I and 1I-1I. In Experiment 3, Group 10D1D differed reliably from Group 1D-1D $(F=35.52, \mathrm{df}=1 / 32, \mathrm{p}<.01)$, while Group 10I-1I did not differ from Group 1I-1I. In both Experiments 1 and 3, these analyses yielded reliable delay effects, with delayed reward conditions running more slowly than immediate reward conditions $(F s=10.0$ and $89.95 ; \mathrm{df}=1 / 20$ and $1 / 36 ; p<.01$, for Experiments 1 and 3, respectively). Delay effects $(\mathrm{F}=55.12, \mathrm{df}=1 / 32, \mathrm{p}<.01)$ as well as (preshift) magnitude effects $(\mathrm{F}=24.5, \mathrm{df}=1 / 36$, $p<.01)$ were also found to be significant at Trial Blocks 3 and 4 of Experiment 3. Both shifted conditions ran more slowly than the unshifted control groups, and both delay groups ran more slowly than the immediate groups.

The slightly greater depression in the $8 \mathrm{D}-1 \mathrm{D}$ as compared with the 8I-1I condition of Experiment 2 was not statistically reliable. Analysis of the data over Blocks 3 and 4 yielded reliable effects of (preshift) magnitude $(\mathrm{F}=6.5, \mathrm{df}=1 / 26, \mathrm{p}<.05)$ and delay $(\mathrm{F}=32.45, \mathrm{df}=1 / 26, \mathrm{p}<.01)$ with slower speeds associated with reward reduction and with delay of reinforcement, but the Magnitude by Delay interaction was not statistically significant.

Finally, analyses of terminal acquisition (A) data yielded significant effects of delay in each experiment $(\mathrm{Fs}=18.48,15.80,29.27$; $\mathrm{df}=1 / 20,1 / 27,1 / 36$; $\mathrm{p}<.01$ ) in Experiments 1, 2, and 3, respectively, with delayed reward conditions running more slowly than immediate reward conditions. Large reward conditions ran faster than small reward conditions in Experiments $1(\mathrm{~F}=11.14, \mathrm{df}=1 / 20, \mathrm{p}<.01)$ and 2 $(\mathrm{F}=4.27$, $\mathrm{df}=1 / 27, \mathrm{p}<.05)$, but not in Experiment 3.

\section{DISCUSSION}

The present results suggest that the extent of the negative contrast effect attending a reduction in reward magnitude is greater when all rewards are delayed rather than delivered immediately following the instrumental response. In conjunction with previous data (e.g., Mellgren, 1971, 1972; Shanab \& Biller, 1972; Shanab \& Cavallaro, 1975), it is thus apparent that "superimposed" delay of reinforcement augments both positive and negative contrast effects resulting from changes in reward magnitude, a circumstance which would appear to be of central relevance to any systematic account of contrast effects.

Any theoretical framework which purports to interpret the facilitative effects of delay of reward on contrast effects should concurrently provide a satisfactory interpretation of contrast phenomena per se. The "ceiling hypothesis" as previously outlined is deficient in both respects. First, while it might provide a convenient format for interpreting the fact that positive contrast is enhanced under conditions of delayed reward, it is inapplicable to the present design and its conceptual counterpart, a "floor hypothesis" (Shanab, Rouse, \& Cavallaro, 1973) is contradicted by the present observation of greater negative contrast in delayed as compared with immediate reward conditions. Secondly, the ceiling hypothesis was, of course, not intended as an interpretation of contrast, but as a supplementary explanatory device (which was) most useful to theoretical approaches to reward-shift effects which predict both positive and negative contrast, e.g., perception-based accounts based on Helson's (1964) adaptation-level theory (cf. Dunham, 1968). Such theories would appear to be deficient in that they neither explain why perceptual differences between large and small reward might be enhanced by delaying rewards, the fact that NCEs occur more reliably than do PCEs under conditions of immediate reward, nor the fact that PCEs when obtained are extended over a substantial number of postshift trials (e.g., Mellgren, Seybert, Wrather, \& Dyck, 1973).

A recent theoretical account of contrast effects within what has been termed a "reinforcement level" approach (Capaldi, 1974, in press) would appear to provide a satisfactory account of both positive and negative contrast effects and the facilitative effects of delay of reward on these phenomena. Within the reinforcement level framework, associative tendencies (habits) are assumed to accrue in "supernormal" amounts when subjects receive greater reward magnitudes than expected as the result of prior reward history, with habits thus accrued providing the basic mechanism responsible for PCEs. Conversely, inhibition is assumed to result when obtained rewards are less than expected rewards, producing NCEs. As Capaldi (in press) has demonstrated in detail, this framework generates the expectation that increases and decreases in both delayed and immediate reward amounts will produce contrast effects. Large PCEs under delayed, as compared with immediate, reward are generated from an interesting variant of the ceiling hypothesis, one with potential application to the present paradigm. According to reinforcement-level theory, various considerations of habit and expectancy yield comparable habit differentials between conditions shifted from small to large reward and large reward controls regardless of the constant conditions of delay. However, since delay increases inhibition which detracts from effective habit strength, and the response is assumed to be a negatively accelerated function of effective habit, it follows that habit differentials between delayed reward groups will yield larger performance differences than will the same differentials between immediate reward conditions. The same reasoning may readily be applied to the present observation that delay of reward enhances NCEs, but only provisionally, since such matters as the manner in which delay-produced inhibition and reward reduction-produced inhibition combine to yield total effective inhibition as well as the relationship of such an assumption to the growth parameter relating response to effective habit remain to be specified.

Finally, as suggested earlier, the facilitative effects of delay of reward on contrast effects produced by changes in reward may be described by the assumption that incentive $(K)$ differences between large and small rewards are more pronounced under longer periods of delay. Such an assumption obviously has little explanatory value unless the fashion in which incentive expectancy values, and changes in these values, may be expected to influence instrumental responding is clearly specified, a matter which we have addressed in a theoretical paper under editorial review (Note 1).

\section{REFERENCE NOTE}

1. McHose, J. H., \& Moore, J. N. An associative, noncontextual theory of instrumental conditioning with application fo the effects of changes in reinforcement conditions. Submitted for editorial comment

\section{REFERENCES}

Bevan, W., \& Adamson, R. Reinforcers and reinforcement: Their relation to maze performance. Journal of Experimental Psychology, 1960, 59, 226-232.

BLACK, R. W. Shifts in magnitude of reward and contrast effects in instrumental and selective learning: A reinterpretation. Psychological Review, 1968, 75, 114-126.

BowER, G. H. A contrast effect in differential conditioning. Journal of Experimental Psychology, 1961, 62, 196-199.

CAPALDI, E. J. Partial reward either following or preceding consistent reward: A case of reinforcement level. Journal of Experimental Psychology, 1974, 102, 954-962. 
Capaldi. E. J. Reinforcement level: $A n$ expectancy-associative approach to relative reinforcement and nonreinforcement effects. (Title unspecified to date). New York: Academic Press (in press).

Dunham. P. J. Contrasted conditions of reinforcement: A selective critique. Psychological Bulletin. 1968. 69. 295-315.

Helson. H. Adaptation-level theon: An experimental and sistematic approach to behavior. New York: Harper and Row. 1964.

McHose. J. H., \& Peters, D. P. Partial reward. the negative contrast effect. and incentive averaging. Animal Learning and Behavior. 1975, in press.

Mellgren. R. L. Positive contrast in the rat as a function of the number of preshift trials in the runway. Journal of Comparative and Physiological Psychology. 1971. 77. 329-336.

Mellgren. R. L. Positive and negative contrast effects using delayed reinforcement. Learning and Motivation, 1972, 3. 185-193.

Mellgren. R. L.. Seybert, J. A.. Wrather, D. M.. \& Dyck. D. G. Preshift reward magnitude and positive contrast in the rat. American Joumal of Psychology. 1973, 86. 383-387.

Shanab. M. E.. \& Biller. J. D. Positive contrast in the runway obtained following a shift in both delay and magnitude of reward. Learning and Motivation, 1972, 3, 178-184.

Shanab. M. E. \& Cavallaro. G. Positive contrast obtained in rats following a shift in schedule. delay, and magnitude of reward. Bulletin of the Psychonomic Society. 1975, 5. 109-112.

Shanab. M. E.. Rouse. L. O.. \& Cavallaro, G. Effects of shifts in delay of reward in rats as a function of reward magnitude. Journal of General Psychology. 1973, 89, 59-66.

(Received for publication July 28, 1975.) 